\title{
Retour sur « Construction et destruction des monuments mégalithiques »
}

\section{Claude Masset}

\section{(2) OpenEdition \\ 1 Journals}

Édition électronique

URL : https://journals.openedition.org/tc/5069

DOI : $10.4000 /$ tc. 5069

ISSN : 1952-420X

Éditeur

Éditions de l'EHESS

\section{Édition imprimée}

Date de publication : 30 juin 2010

Pagination : 450-452

ISSN : 0248-6016

\section{Référence électronique}

Claude Masset, «Retour sur « Construction et destruction des monuments mégalithiques » », 


\section{Claude Masset}

CNRS, GDR Méthodes d'études des sépultures
Cultures matérielles 2 - VII

Techniques \& Culture 54-55, volume 2, 2010 : 453-469

\section{CONSTRUCTION ET DESTRUCTION DES MONUMENTS MÉGALITHIQUES}

En présence d'un menhir, d'un dolmen, d'un monument « mégalithique » quel qu'il soit, le touriste intrigué se demande volontiers comment l'architecte néolithique, considéré comme "primitif », pouvait faire ériger des blocs aussi énormes. À la limite, certains vont jusqu'à imaginer des sortes de sauvages hirsutes assistés - sinon supplantés par quelque Extraterrestre...

\section{Construction des mégalithes}

Excluant a priori pareilles extravagances, il faut bien reconnaître que les témoignages archéologiques concernant la construction des mégalithes sont des plus maigres, sauf en ce qui concerne la façon de se procurer les pierres qui les constituent.

\section{Extraction}

Des carrières ont été retrouvées, notamment à Bougon dans les Deux-Sèvres (Mohen 1989), où se trouvaient encore des pics en bois de cerf ayant pu servir à attaquer les fissures de la roche, ou à en élargir les plans de stratification. L'utilisation expérimentale de pics, réalisés dans un même matériau, a montré que cette hypothèse était probable, d'autant 


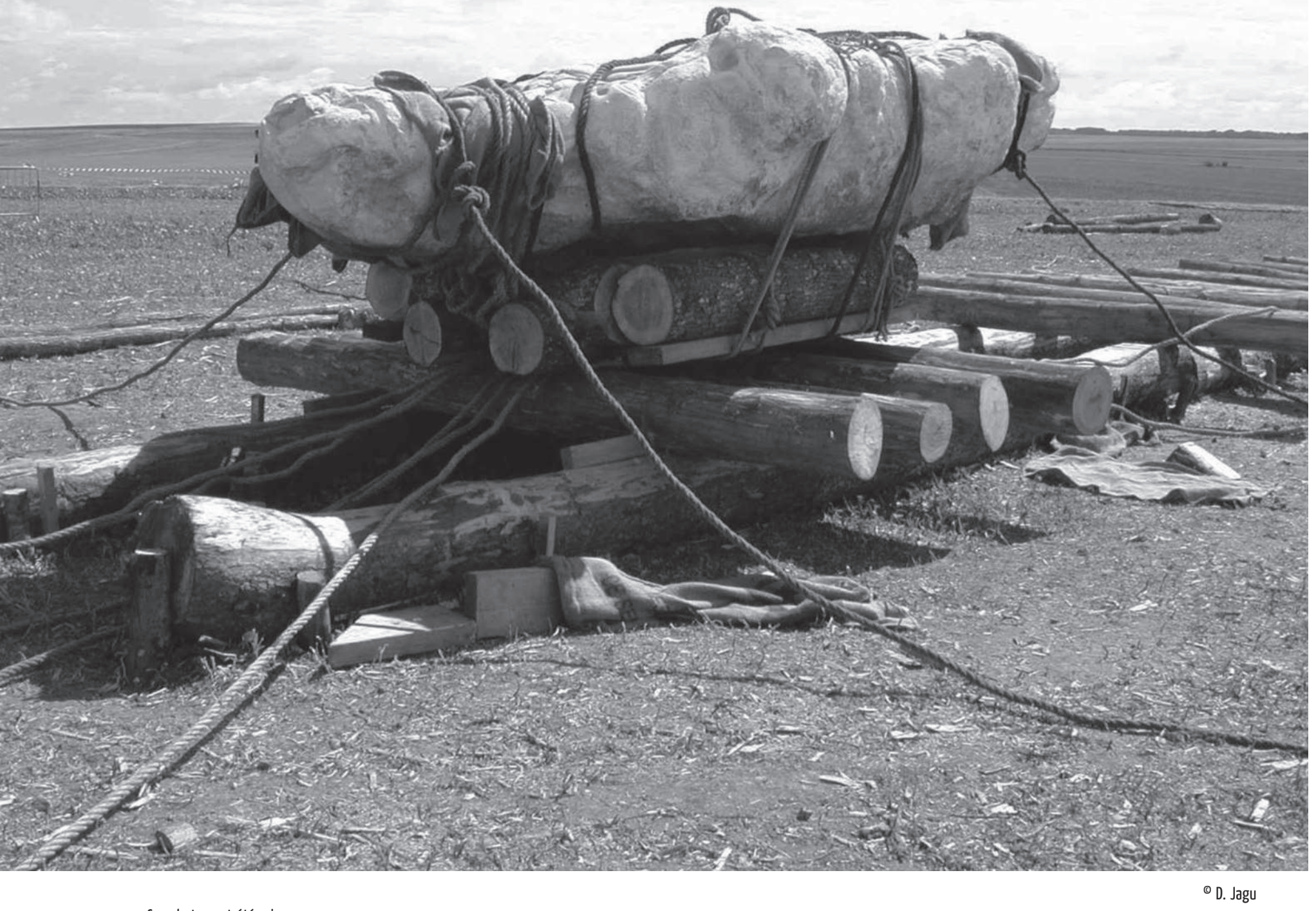

Ces photos ont été prises

en l'an 2000. La commune de Mévoisins, dans l'Eure-et-Loir, avait décidé de marquer le changement de millénaire par l'érection d'un menhir, laquelle érection devait se faire exclusivement par des moyens accessibles au Néolithique. C'est plus pittoresque quïnstructif. Cela dit, ces photos ne font pas double emploi avec ce qui peut avoir été publié par ailleurs dans ce domaine, depuis que des gens s'intéressent à la question.

que les outils expérimentaux présentèrent des traces d'utilisation de même nature et de même localisation que leurs homologues archéologiques. Sans doute fallait-il, pour atteindre une efficacité maximale, une connaissance approfondie du grain de la pierre, et plus généralement des particularités de la roche envisagée.

Trouvés également sur les lieux, des percuteurs en chaille ou en silex ont pu servir à enfoncer les pics dans la roche, ou à la mettre en forme par « bouchardage ». Cette technique consiste à frapper à petits coups la surface qu'on veut modifier, pour en détacher peu à peu de la poussière ou de menus éclats. À condition de disposer du temps nécessaire, on arrive ainsi à façonner les roches les plus dures, voire à les détacher de leur carrière originelle. Pareille technique, qui laisse sur les surfaces traitées des traces reconnaissables, est attestée aussi bien dans l'Égypte antique et à l'île de Pâques, que dans l'Europe néolithique. Enfin à Bougon une omoplate d'aurochs, ailleurs des omoplates de bœuf, servaient apparemment de pelles pour l'enlèvement des déblais. 


\section{Transport et érection}

La façon dont les pierres étaient véhiculées depuis leur lieu d'extraction n'a laissé, par contre, à peu près aucune trace archéologique. Le paysage à travers lequel se fit ce transport a changé plusieurs fois depuis les quelque cinq ou six mille ans qui nous séparent de ces opérations architecturales, et il n'en a donc gardé aucun témoignage. L'érection d'une dalle nécessitait probablement des échafaudages, mais leurs auteurs n'avaient aucune raison de les conserver, et de toute façon le bois sans doute utilisé à cet effet n’aurait pas subsisté. On note souvent la dissymétrie du trou d'implantation: un menhir ne se fichait pas dans le sol comme une météorite. Il était, selon toute apparence, amené en position horizontale, puis peu à peu relevé. On s'en serait douté. Dans un cas au moins, à SaintePallaye dans l'Yonne, il restait trace de madriers horizontaux ayant supporté, avant son érection, les cinq tonnes d'une dalle; ils auraient été progressivement détruits par le feu afin de faciliter le basculement de cette grosse pierre (Carré 1963).

Sevré de documentation archéologique, on est donc réduit à des comparaisons et à des simulations.

Guère plus récentes que nos érections néolithiques, des gravures égyptiennes ou mésopotamiennes montrent les techniques employées alors en Orient. On voit, par exemple, un gigantesque monolithe solidement arrimé à une sorte de traîneau de bois auquel sont fixées des cordes. Sous la direction d'un personnage installé sur le traîneau, beaucoup d'hommes tirent sur ces cordes (peu importe leur nombre: il dépend surtout des dimensions de la gravure). Autour d'eux s'activent des porteurs d'eau qui viennent mouiller le sol devant le traîneau, des musiciens appelés à rythmer et à coordonner l'effort, des porteurs de fouets chargés sans doute de rappeler aux participants les inconvénients de la paresse. Pour nous modernes, ils rappellent surtout à quel genre de société nous avons affaire. Dans cet Orient classique où naît l'État, la paysannerie est déjà serve: convoquée après la moisson pour servir le roi, elle n'attend de lui que le pain quotidien pendant la durée des travaux. Rien n'oblige à penser qu'il en était de même dans notre Occident néolithique où d'autres indices suggèrent au contraire une hiérarchie sociale encore discrète: la présence des « pères fouettards » n'y est donc point assurée. L'importance de la main-d'œuvre n'implique pas nécessairement une très grande dimension des collectivités locales: comme autrefois d'une ferme à l'autre en temps de battage, ou d'un village à l'autre, on pouvait s'entraider, à charge de revanche, naturellement. D'aussi grandes réunions n'allaient sans doute pas sans festivités, sans dépenses d'alimentation pour le moins. La communauté demanderesse (ou l'autorité responsable) ne pouvait guère se dispenser de nourrir ses invités, et y voyait même sans doute une source supplémentaire de prestige, dans la mesure où la quantité de nourriture, mesure de l'abondance de la main-d'œuvre, était elle-même un reflet de l'immensité de la tâche envisagée.

Un fait divers survenu à Paris en 1818 vient compléter ces observations historiques: le transport de la statue d'Henri iv jusqu'au square du Vert-Galant. Fondue aux dépens de plusieurs statues ayant représenté notamment « l'usurpateur » déchu, Napoléon, elle n'atteignait pas moins de vingt-cinq tonnes; et même quarante si l'on ajoute son traîneau et son dispositif de protection. Pour la remorquer on avait prévu dix-huit paires de bœufs. Laffaire faillit pourtant mal tourner car, s'il est difficile de mettre en mouvement une lourde charge, il n'est guère plus facile de l'arrêter une fois lancée lorsqu'elle prend une mauvaise direction. Cette lourde masse allait verser, quand la foule des badauds, s'emparant des cordes, procéda avec succès au remorquage. La popularité d'Henri IV y était-elle pour 
quelque chose? Aurait-on obtenu le même résultat s'il s'était agi de Louis xviI ou même de Louis XIv? Toujours est-il que la foule manifestait, paraît-il, un certain enthousiasme (Lafolie 1819). Cet exemple montre qu'il importe de pouvoir s'adapter sans délai à des situations susceptibles de changer brusquement, ce qui met en lumière l'intérêt de la main-d'œuvre humaine, de préférence à la traction animale.

Des observations ethnologiques permettent de compléter ces exemples, et aussi de mettre en avant d'autres possibilités. Comme dans l'Égypte antique, l'usage du traîneau est attesté en divers endroits: à Madagascar, par exemple, au siècle dernier (Joussaume et Raharijaona 1985). On sait qu'il fallait alors régulariser le sol sur le parcours prévu, ce qui semble avoir été le cas dans certains de nos sites néolithiques européens.

Utilisée en Asie du Sud-est, la technique du panglong permet le transport de billots pouvant peser plusieurs tonnes (Garfitt 1979). On dispose, côte à côte, sur la longueur du parcours prévu, deux lignes parallèles de troncs d’arbres sommairement ébranchés, dont la surface supérieure a été aplanie. Des traverses, assemblées à mi-bois, reposent sans faire de saillie sur ces rails. Fixé à une sorte de traîneau en bois dur, le bloc à déplacer peut être halé avec, paraît-il, peu d'effort, surtout si l'on enduit de graisse la surface supérieure du panglong. Ce dispositif est réutilisable, avantage appréciable s'il dessert une carrière susceptible de fournir plusieurs blocs. Un autre procédé, observé en 1991 par Anne Debut dans l'île indonésienne de Sumba, consiste simplement à porter la pierre à dos d'hommes, à l'aide d'un système de cordes et de perches. D'après ce témoignage il fallait dix-neuf hommes pour environ une tonne (communication personnelle). Il est à noter que, pour des poids plus élevés, les habitants de Sumba connaissent aussi l'usage du traîneau: il y est attesté il y a un quart de siècle pour une dalle de onze tonnes, déplacée sur trois kilomètres; on avait utilisé à cet effet les efforts de 552 personnes (Heizer 1966).

Parallèlement à ces exemples historiques ou ethnologiques ont été tentées des simulations. On peut sans doute assimiler à une simulation une observation réalisée pendant la Première Guerre mondiale par un préhistorien allemand, le professeur Jacob-Friesen. Alors commandant d'artillerie, il avait à faire transporter des canons lourds en terrain difficile. Pendant l'hiver, quand la terre était durcie par le gel, il réussissait en trois jours ce qui lui aurait demandé, écrit-il, quatre semaines en été. Appliquant cette observation au transport des mégalithes, il pensait que cette opération aurait grandement profité des conditions hivernales, saison pendant laquelle, précisément, les paysans sont libérés des travaux des champs. Dans un tel contexte, de simples leviers auraient alors suffi à déplacer une grosse pierre par très courtes distances, en un cheminement qu'on pourrait assimiler à des sauts de puce.

Intéressante, cette idée n'est pas envisageable sous tous les climats; ne serait-ce qu'au Portugal dont les dolmens comptent parmi les plus anciens d'Europe. Des expériences à base de traîneaux ont été réalisées à plusieurs reprises et dans divers endroits: dans l'île de Pâques par exemple (Heyerdahl 1958); dans les environs de Stonehenge en Angleterre, où l'essai fut complété par la traversée d'un cours d'eau sur une sorte de radeau (Atkinson 1956). L'expérience la plus notoire, tant par la masse du monolithe en jeu que par la technique et le type de main-d'œuvre utilisés, est celle que réalisa Jean-Pierre Mohen en 1979 à Bougon dans les Deux-Sèvres. Appliquant une suggestion émise dès le siècle dernier par le roi archéologue Frédéric viı de Danemark, il fit construire, en utilisant à cette fin des haches polies, une sorte de chemin de halage semblable à un panglong dont les traverses auraient été remplacées par des troncs d'arbres de section circulaire servant de rouleaux. À l'aide de cordes d'une technologie également néolithique, l'archéologue 
réussit ainsi, avec l'aide d'environ 200 habitants d'un village voisin, à déplacer une masse de béton pesant trente-deux tonnes. Les troncs libérés à l'arrière par le mouvement du bloc étaient replacés à l'avant; quand le bloc tendait à dévier de sa course, de simples leviers suffisaient à le remettre dans le droit chemin. L'un des enseignements les plus intéressants de cette expérience est l'espèce d'atmosphère ludique dans laquelle elle s'est passée, le plaisir de réaliser ensemble une action sortant de l'ordinaire (Mohen 1989 : 176 sq.).

À mes yeux, l'aspect le plus instructif de ces tentatives est le contraste entre leur diversité... et leur même succès. Somme toute, on peut s'y prendre comme on veut: dès qu'on est capable de réunir un

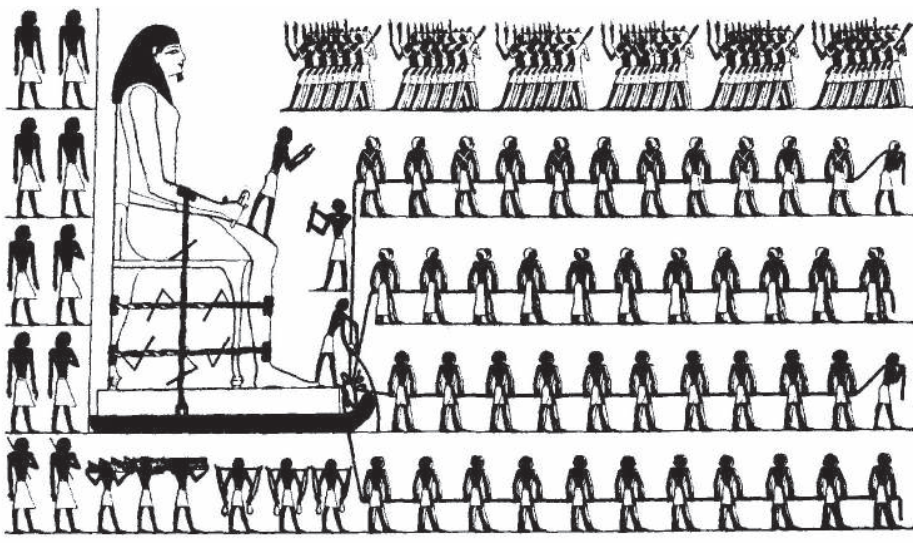
assez grand nombre d'hommes (d'hommes, non d'animaux de trait, trop peu maniables), et qu'on a une idée claire de ce qu'on veut réaliser, cela marche. La même constatation peut être faite à propos du relèvement des blocs tombés: que ce soit à l'aide de leviers permettant de glisser sous la pierre des petits cailloux, comme le fit Thor Heyerdahl dans l'île de Pâques (1958), ou par divers types d'assemblages de madriers permettant, grâce à des cordes, de modifier la direction des forces appliquées au monolithe (Coles 1973), celui-ci finit toujours par se redresser. Ajoutons que nos ancêtres néolithiques disposaient à coup sûr de traditions et de tours de main leur facilitant la tâche. Chacun des exemples précités ne saurait donc être qu'une suggestion, dont la réalité pouvait d'autant plus s'écarter que les conditions étaient plus variables. L'emploi du panglong en Asie, celui de rondins à Bougon, ne sont attestés que pour des masses ne dépassant pas quelques dizaines de tonnes. On peut douter nous le verrons plus loin, qu'ils aient été bien adaptés à des charges beaucoup plus importantes. Or une dalle de « La Cueva de Menga » à Antequera en Andalousie pesait quelque 180 tonnes; le grand menhir brisé « Men er Hroek » de Locmariaquer dans le Morbihan atteignait près de 350 tonnes. Reste alors le traîneau dont l'expérience montre qu'il peut supporter plusieurs centaines de tonnes.

La mise en place des dalles de couverture sur des piliers verticaux pose des problèmes un peu différents. On pouvait, par exemple, remorquer la dalle le long d'un plan incliné réalisé à cet effet, procédé attesté tant dans l'Égypte ancienne qu'à Sumba en 1966. Dans notre Néolithique européen, un tel plan incliné aurait pu constituer déjà une partie du tumulus destiné plus tard à enrober le dolmen. Une variante de ce procédé, d'une étonnante économie de moyens, a été imaginée en Tchécoslovaquie en 1991. La petite ville de Strakonice ayant décidé de s'offrir une réplique en béton d'un des trilithes de Stonehenge, utilisa délibérément à cet effet des moyens à la portée d'une population néolithique. Sous la direction du professeur Pavel fut construit un plan incliné qui n'était formé que de deux troncs de chêne, longs de dix mètres, soigneusement écorcés et enduits de graisse; leur extrémité supérieure reposait à 3 mètres 90 de hauteur, sur les sommets des orthostates préalablement érigés. Pour remorquer, sur cette pente de quelque 25 degrés un linteau qui pesait plus de cinq tonnes, des cordes le reliaient, par-delà les orthostates, à deux leviers également en chêne, longs de 4 mètres 50 pour un diamètre de 25 centimètres. Grâce à ce procédé renouvelé d'Archimède, dix hommes seulement, pas un de plus, réussirent ainsi à hisser le linteau et à l'installer sur son emplacement définitif. 


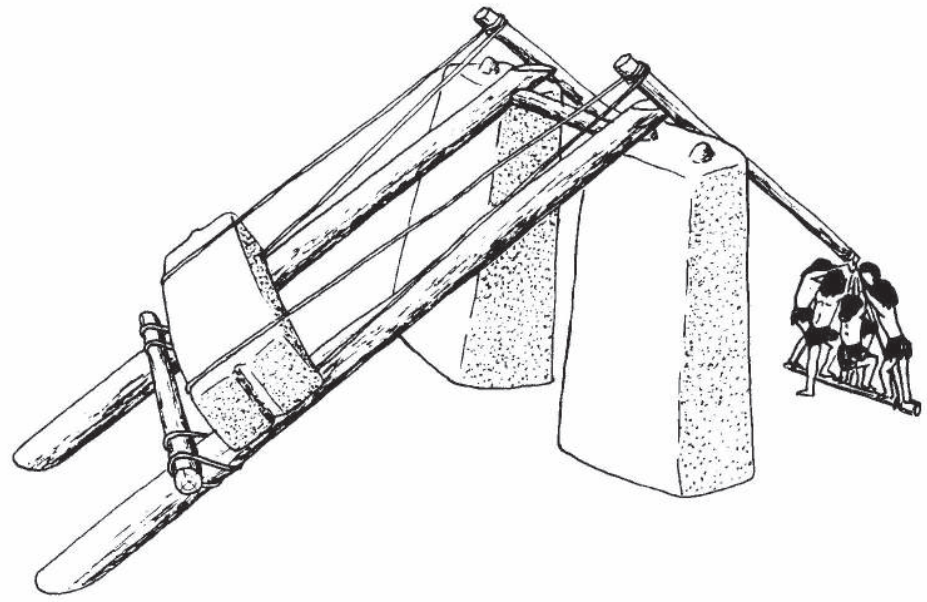

Une expérience exécutée en République Tchèque, à propos de l'érection des mégalithes de Stonehenge.
Sans plan incliné et de façon plus rustique, J.-P. Mohen utilisa pour un projet analogue un édifice formé de barres de bois posées à angle droit les unes sur les autres. On soulevait à l'aide de leviers, tour à tour, chacune des extrémités du bloc qu'on voulait hisser; on glissait sous elles d'abord des cales, puis des barres supplémentaires. En pareil cas, la mise en place des orthostates aurait donc pu être réalisée après l'arrivée sur les lieux de la dalle de couverture, à travers la charpente qui l'aurait soutenue... À ma connaissance, personne n’a essayé.

\section{Témoignages de compétences techniques}

Si la fouille est peu loquace sur la façon de construire les monuments mégalithiques, elle fournit en revanche des indications sur les sociétés qui s'exprimaient à travers leur érection. Ces lourdes pierres furent mises en place avec une certaine économie de moyens. Un menhir n'est guère enfoncé dans le sol de plus du cinquième de sa hauteur. Il est solidement maintenu par des « blocs de calage » qui prennent appui à la fois sur sa surface et sur les parois de la fosse dans laquelle il est implanté, système qui permet au mégalithe d'affronter les millénaires. Dans beaucoup de dolmens et d'allées couvertes, des piliers verticaux, dits « orthostates », furent trouvés en position oblique. Que cette disposition ait été ou non voulue par le constructeur, force est de constater qu'elle résista pendant bien des siècles aux sollicitations de la gravité. Certains nivellements paraissent avoir été de bonne qualité. À Méréaucourt (Somme), dans une allée couverte dont nous reparlerons, la surface supérieure d'une couche de condamnation est tout à fait horizontale, alors que celle du sol environnant est déclive. Un autre exemple est fourni par des monuments dont la couverture mégalithique est composée d'énormes dalles qui reposent sur des murettes en pierres sèches: ce résultat suppose une bonne horizontalité de la surface supérieure des murettes. Il serait vain d'espérer retrouver les instruments dont se servaient à cet effet les Néolithiques. Pour les besoins du nivellement, l'Antiquité classique utilisait de longues planches soigneusement aplanies sur lesquelles on versait de l'eau; quand celle-ci ne coulait plus d'aucun côté, on savait que l'horizontalité était atteinte. Si rustique soit-il, cet instrument suffisait aux exigences, pourtant rigoureuses, de l'édification des aqueducs; sans doute fallait-il un entraînement qui ne s'acquérait pas en un jour. Peut-être peut-on créditer les Néolithiques d'un outillage comparable.

\section{Témoignages d'une organisation sociale}

De toute façon, on ne saurait douter que l'érection d'un monument mégalithique n'ait exigé une autorité, politique ou religieuse, capable d’assurer la coordination nécessaire. Elle suppose aussi la présence d'experts, dépositaires des traditions techniques: un architecte qui sache maîtriser le transport de lourdes pierres et pour qui leur mise en 
place n'eût point trop de secrets; sans doute aussi des cordiers, des charpentiers, des géomètres peut-être, voire des musiciens. La société qu'évoque la présence de tous ces techniciens n'est pas des plus simples. Ces hypothèses en recoupent d'autres faites à partir d'observations réalisées sur des squelettes de la même époque. L'existence de trépanations réussies, même dans des parties de la boîte crânienne où ce genre d'opérations est connu comme particulièrement délicat, indique que ces antiques communautés rurales n'étaient pas exclusivement agraires, et qu'elles incluaient aussi des spécialistes de bon niveau.

\section{Destruction des mégalithes}

Peu prolixe sur la façon dont étaient érigés les monuments mégalithiques, la fouille se montre plus loquace en ce qui concerne leur destruction. La plupart d'entre eux furent en effet trouvés ruinés, leurs orthostates renversés, leurs tables effondrées ou manquantes, leurs tumulus disparus. Il était autrefois d'usage d'attribuer ces dégradations aux outrages du temps, à une maladresse éventuelle des constructeurs de mégalithes, à l'action d'une Église médiévale soucieuse d'extirper les dernières traces du paganisme, à des prélèvements opérés en vue d'empierrer les routes ou d'édifier à bon compte les habitations du voisinage. Sans sous-estimer l'importance de ces derniers types de destruction, mettons hors de cause les architectes néolithiques: les fouilles les plus récentes ont clairement fait apparaître leur compétence. Elles ont montré surtout qu’une partie des dégradations les plus anciennes étaient tout à fait intentionnelles.

\section{Destructions en cours d'utilisation}

À la Chaussée-Tirancourt (Somme), nous sommes en présence d'un monument mégalithique érigé au fond d'une fosse: une sorte d'allée couverte dépourvue de couverture, dont les orthostates les plus grands n'atteignent pas le niveau du sol environnant. On peut voir sur le plan qu'il manque deux orthostates dans la zone d'entrée (figure 1). La fouille a montré que ceux-ci avaient jadis été présents: à leur emplacement, la craie picarde était entaillée et présentait des traces d'écrasement; par ailleurs il s'y trouvait encore en place un fragment d'orthostate ainsi que quelques blocs de calage. L'une de ces empreintes était fossilisée sous deux couches d'inhumations indubitablement néolithiques. L'extraction de l'orthostate correspondant datait donc, sinon des touts débuts, du moins d'une époque ancienne de l'utilisation du monument (Leclerc et Masset 1980). Des constatations de même ordre ont été effectuées par Henri Duday lors de la fouille du dolmen de Villedubert dans l'Aude (inédit).

Après une première période dans l'utilisation funéraire de ce sépulcre, des orthostates en furent extraits, d'autres y furent brisés; une partie des débris servit de plancher pour une nouvelle période sépulcrale.

Si le caractère délibéré d'une dégradation est évident dans le cas d'une extraction d'orthostate, en est-il de même quand un pareil bloc est trouvé simplement basculé? la fouille d'une autre allée couverte dans la Somme permet de répondre affirmativement. À Méréaucourt, sur les os d'un premier niveau d'inhumations (couche V) fut déposée une 


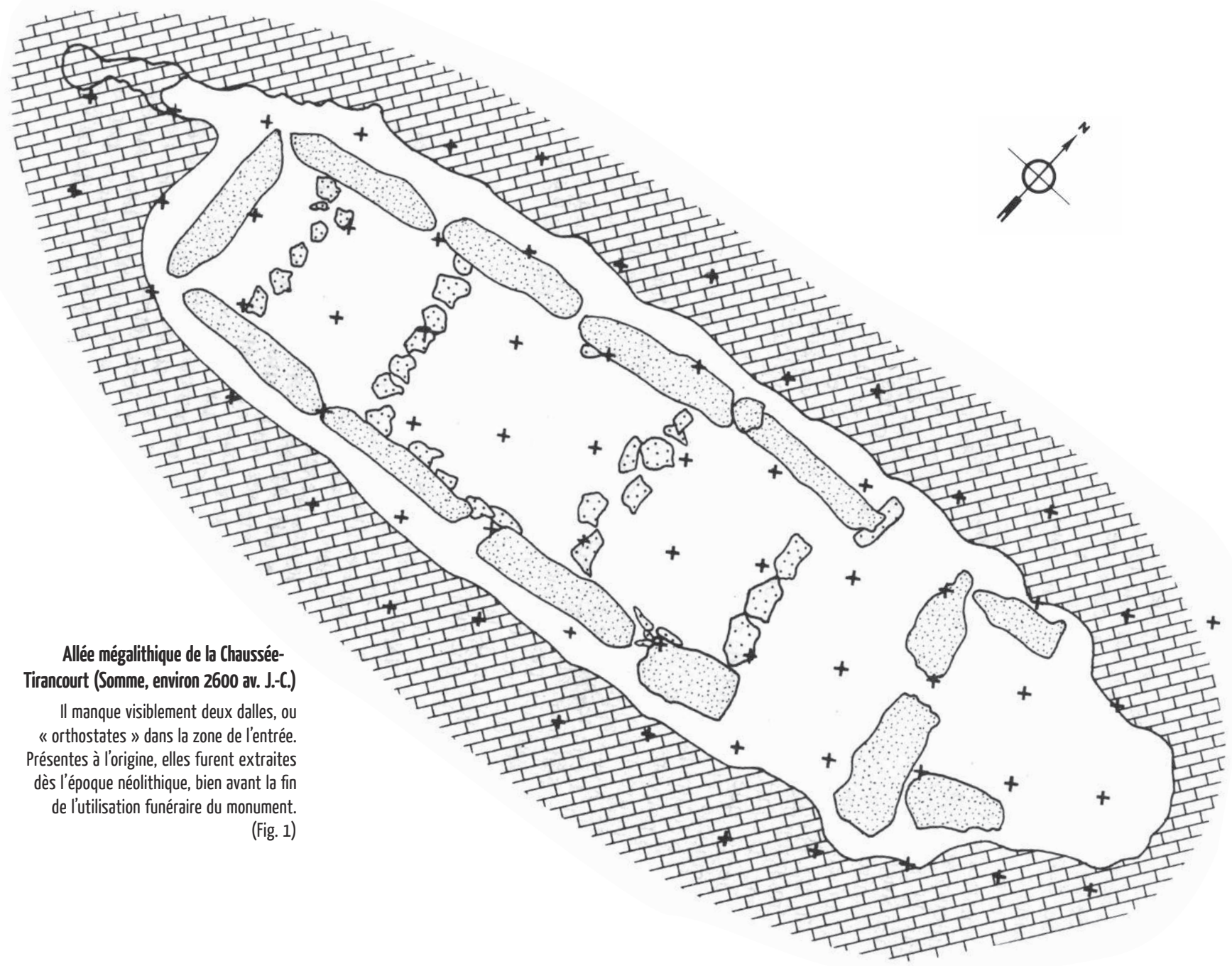

mince couche de terre et de cailloux (couche IV), sur laquelle bascula un orthostate. Les inégalités de sa surface vinrent s'imprimer sur la couche IV, et même à travers elle sur le sommet de la couche V. Ce bloc servit ensuite de limite aux inhumations de la couche III. Suggéré par la chronologie, le caractère intentionnel de cette chute est confirmé par la technique qu'ont employée pour ce faire les Préhistoriques. Erigé au fond d'une petite fosse, sorte de « rail d'implantation », un orthostate tient debout, nous l'avons vu, par le jeu d'éléments plus petits, blocs de calage, qui le coincent solidement. À Méréaucourt, du côté où avait basculé cet orthostate, il n'y avait pas, il n'y avait plus de blocs de calage: ils avaient été enlevés avant l'arrivée de la couche III, plus précisément alors que les Néolithiques mettaient en place la couche IV. À Guiry-en-Vexin (Val d'Oise), l'allée couverte de « La Ferme Duport » est en partie hypogée: l'extrémité la plus éloignée de l'entrée se trouve, sur une longueur de plusieurs mètres, creusée dans la roche. On y a constaté que la couverture ainsi constituée s'effondra un beau jour, entraînant dans sa chute les orthostates du fond, sans interrompre pour autant la succession des inhumations. Les orthostates de la zone d'entrée s'effondrèrent d'ailleurs aussi, à une époque indéterminée, sans que s'interrompe non plus la succession des inhumations (Peek 1975 : 86-87). Si ces phénomènes n'avaient été que de simples accidents, on ne pourrait qu'admirer le 
courage des fossoyeurs qui fréquentaient sans faiblir un monument manifestement des plus dangereux! Je serais plutôt tenté d'y voir des déprédations volontaires, semblables à celles observées dans les monuments précités. La publication définitive de la fouille de «La Ferme Duport » permettra peut-être de mettre en relations ces accidents, que je crois intentionnels, avec des variations dans la stratigraphie, et peut-être de préciser la technique employée à cette fin par les Néolithiques.

Nous pouvons donc observer ces gestes de destruction partielle alors que se poursuivait l'utilisation du monument: le basculement d'un orthostate jouait apparemment, à moindre frais, le même rôle qu'une extraction. Cette technique a pu être générale et serait alors une des causes de l'état misérable dans lequel ont été trouvés tant de dolmens. Si nous ne sommes pas en mesure de retrouver tout le sens de ces déprédations, nous pouvons toutefois constater, chaque fois qu'a été suffisante la précision de la fouille, qu'elles sont liées au passage d'une phase d'utilisation à une autre.

\section{Destructions en fin d'utilisation}

Les destructions intervenant en fin d'utilisation bouleversent de larges surfaces. Dans un petit nombre de cas leur caractère néolithique a pu également être mis en évidence. L'exemple de la Chaussée-Tirancourt est particulièrement démonstratif. Après le dépôt du dernier corps, une couche de limon mêlée de pierres vint uniformément recouvrir le monument et ses abords, laissant toutefois dépasser le sommet des orthostates. Un certain temps s'écoula. Un jour, vers 1800 av. J.-C., vinrent des gens qui, autour de chaque orthostate creusèrent des fosses et allumèrent de grands feux. Sous l'effet de la chaleur, le grès landénien éclata en milliers d'écailles brûlées, retrouvées sur les lieux. La plupart des orthostates perdirent alors leurs sommets. Le caractère préhistorique de cette opération est attesté non seulement par le radiocarbone, mais aussi par la présence de percuteurs en silex mêlés aux éclats de grès.

La technique utilisée n'est pas connue dans le détail. Les Préhistoriques ont pu jouer sur les différences de température entre les deux extrémités d'un même bloc de grès. Une expérience, réalisée en 1974 à la Chaussée-Tirancourt par Philippe Andrieux, a montré qu'un feu très vif attisé par un courant d'air bien dirigé, pouvait casser un bloc de grès landénien de cinquante kilos, à condition que la chaleur n'affecte qu'une seule extrémité du bloc. C'est pourtant autour de chaque orthostate que s'observent les traces d'incendies préhistoriques. Il y a bientôt trois siècles, J. Aubrey décrivait la façon dont des paysans détruisaient par le feu les énormes menhirs de grès du grand cromlech d'Avebury dans le Wiltshire (cité par Burl 1979 : 44). Après avoir renversé un de ces blocs, ils allumaient un grand feu le long de la future ligne de fracture. Une fois atteinte la température voulue, ils arrosaient le menhir d'eau froide et aussitôt après frappaient l'emplacement désiré d'un coup de marteau. Si intéressantes que soient ces expériences, elles s’appliquent mal à la Chaussée-Tirancourt où les écailles du grès avaient perdu toute qualité mécanique: on peut les casser entre les doigts. Plutôt qu'à une récupération de matière première, comme à Avebury s'agissait-il seulement de diminuer artificiellement la hauteur des orthostates, afin de pouvoir inhumer le monument dans sa totalité et de récupérer ensuite, à des fins agricoles, la surface occupée? L'exemple de Méréaucourt, distant seulement d'une trentaine de kilomètres, incite à exclure cette dernière hypothèse. Là aussi le monument fut presque entièrement réenfoui, mais par un ensemble de dalles et de rognons de silex qui 
en rendait la superficie presque totalement infertile. Nous avons affaire, semble-t-il, à une destruction symbolique. Si le but recherché pouvait être de marquer spectaculairement la fin d'une époque, il paraît avoir été plutôt de soustraire ce qui restait du monument aux regards des hommes.

Un geste analogue s'observe à Changé (commune de Saint-Piat), près de Maintenon dans l'Eure-et-Loir, sous une couche de condamnation assimilable à celles de la ChausséeTirancourt et de Méréaucourt. Mais ici, nulle trace de feu. Une dalle de couverture y fut cassée en plusieurs morceaux dont l'un, mesurant près de sept mètres carrés, fut transporté à quelques mètres au sud-ouest du monument; la plupart des autres fragments avaient disparu. Un orthostate avait basculé. Les sommets des autres avaient été attaqués à coups de percuteurs; par endroits sont même observées les traces de "boîtes de débitage ", procédé employé par les carriers pour briser les pierres. Il consiste à introduire dans des trous relativement peu profonds, mais de forme allongée, des coins de bois bien secs, qu'on fait ensuite gonfler à l'eau. Leur force se révèle souvent suffisante pour déterminer des lignes de fractures, à condition, là encore, de bien connaître le grain de la pierre. L'existence d'anciennes fouilles intempestives empêche d'affiner à coup sûr la position stratigraphique de ces « boîtes de débitage ». Certaines d'entre elles, pourtant, paraissent bien avoir été recouvertes par la couche de condamnation du monument, couche dont le caractère néolithique ne fait aucun doute, quoiqu'elle ait été, hélas, cruellement amputée en 1924. Ajoutons qu'un premier cairn englobant le dolmen avait été entièrement démonté avant la succession de ces diverses opérations (Jagu et Van Vliet 1991). Un autre dolmen de Changé, dit du « Berceau », a gardé sa dalle de couverture, mais celle-ci est fracturée; elle présente des «boîtes de débitage » qu'on ne peut malheureusement pas dater. La fouille a montré qu'un des morceaux de ce bloc énorme, basculé, repose sur le dallage de fond de la chambre funéraire: celle-ci était donc vide quand il est tombé. Encore vide (donc néolithique)? - Déjà vidée (très soigneusement)? il n'est pas possible d'en dire davantage; mais l'exemple de son voisin, dont la dalle partit en morceaux au début du quatrième millénaire avant notre ère, suggère là aussi un geste très ancien.

Nous n’avons pas à Méréaucourt de trace certaine de telles déprédations intentionnelles. Une des deux dalles de couverture y fut toutefois trouvée brisée en quatre morceaux, restés sur place. Ancienne, sa fracture ne peut malheureusement pas être datée avec quelque précision; la roche est de médiocre qualité et aucune « boîte de débitage » n’a été retrouvée. L'autre dalle, épaisse de près d'un mètre, aurait été difficile à casser; mais elle avait basculé dans la tombe, et cela d’une bien curieuse manière. Débordant largement la paroi méridionale de l'allée couverte, elle n'en atteignait pas la paroi septentrionale (figure 2)! Selon toute apparence, à une époque qu'il n'est pas possible de déterminer, on l'avait fait riper en direction du sud, jusqu'à ce qu'elle basculât. Il ne s'agit pas ici, non plus qu'à la Chaussée-Tirancourt, de récupérer un matériau convoité, et ce travail a exigé évidemment l'intervention d'un grand nombre d'hommes. On est donc tenté de voir dans ces deux formes différentes de déprédation la manifestation d'une même intention, celle d'abaisser un monument qui ne servait plus pour l'usage funéraire, afin de le soustraire, ainsi que je l'ai suggéré, à l'attention des vivants.

Ces gestes de destruction volontaire nous surprennent surtout dans la mesure où ils s’adressent à des monuments mégalithiques, édifices par nature très résistants. Mais il y a sans doute lieu de rapprocher ces dégradations des incendies en fin d'utilisation, bien attestés dans les sépultures collectives non mégalithiques de Grande-Bretagne connues sous le nom de long barrows. Ces monuments étaient des sortes de « maisons des morts » 
en bois, auxquelles, un beau jour, on mettait le feu. Le fait que leurs décombres étaient par la suite recouverts de plusieurs milliers de tonnes de sédiments, atteste du caractère non accidentel de l'opération.

L'exemple de déprédation le plus célèbre et le plus frappant est celui d'un immense menhir orné de gravures qui se dressait jadis, sur quelque quatorze mètres de hauteur, au voisinage du golfe du Morbihan. Il pouvait peser une centaine de tonnes. Un de ses morceaux constitue aujourd'hui la dalle de couverture du dolmen des Marchand à Locmariaquer; un autre recouvre celui de Gavrinis à cinq kilomètres du premier, dans la commune de Larmor-Baden: les gravures de l'une continuent exactement celles de l'autre. Un troisième fragment, quoique dépourvu d'ornementation, paraît bien former la table d'un autre dolmen de Locmariaquer, au sein du long tumulus dit «Er Vringlé ». Qu’on ait réutilisé, faute de mieux, les éléments d'une stèle abattue accidentellement est une hypothèse envisageable, d'autant plus qu'on n'y voit nulle trace de « boîte de débitage ». Mais le cas n'est pas isolé. Sans quitter les parages du golfe du Morbihan, on peut citer plusieurs autres exemples de réemplois comparables: « Les idoles qu'on abat », titrait déjà J. L'Helgouach en 1983, avant même la découverte du « recollage » entre Gavrinis et Locmariaquer. Le caractère incontestablement volontaire de certaines autres destructions, comme celles évoquées plus haut à Villedubert et à la Chaussée-Tirancourt, invite certes à voir également un caractère intentionnel dans les dégradations prodigieuses observées autour du golfe du Morbihan; mais la question n'est pas réglée. Un jeune chercheur, Eric Gaumé, familier du travail de la pierre, se montre réservé dans une thèse encore inédite. Les fractures, remarque-t-il, ne présentent pas trace de travail humain, et ne suivent pas les plans de clivage naturel de la pierre; elles se seraient plutôt produites lors de la chute des stèles, et cela à des emplacements non prévus à l'avance. Il observe par surcroît que ces immenses mégalithes sont tous tombés dans la même direction: l'hypothèse d'un cataclysme suivi de réemploi ne lui paraît donc point à exclure (Gaumé).

Il faudrait être en mesure de citer un bien plus grand nombre de sites. La médiocre qualité des anciennes fouilles, qui constituent hélas la grande majorité de nos documents, ne le permet malheureusement pas. Sans vouloir sous-estimer l'étendue des déprédations plus récentes, les éléments dont nous disposons permettent du moins d'affirmer que les Néolithiques aussi cassaient volontairement leurs propres monuments, tant en cours d'utilisation, qu'au moment où ils décidaient d'en terminer l'usage. Les techniques employées à cette fin ont pu être reconnues dans un petit nombre de cas: éclatement par le feu à la Chaussée-Tirancourt, percussion à coups de pierre dure dans ce dernier

\section{Allée couverte mégalithique de Méréaucourt (Somme)}

Un orthostate, « F15 », renversé sur la plus ancienne des deux couches d'ossements humains, resta dans cette position alors que les inhumations continuaient dans le sépulcre.

La dalle de couverture « $\mathrm{H} 15$ » fut trouvée brisée en place. Sa voisine « $G 12$ » n'atteignait plus les orthostates « H13 » et « J12 » qui, normalement, eussent dû lui servir d’appui.

Elle fut trouvée basculée à l'intérieur de la chambre funéraire; de l'orthostate « H13 » il ne restait qu'un moignon. (Fig. 2) 


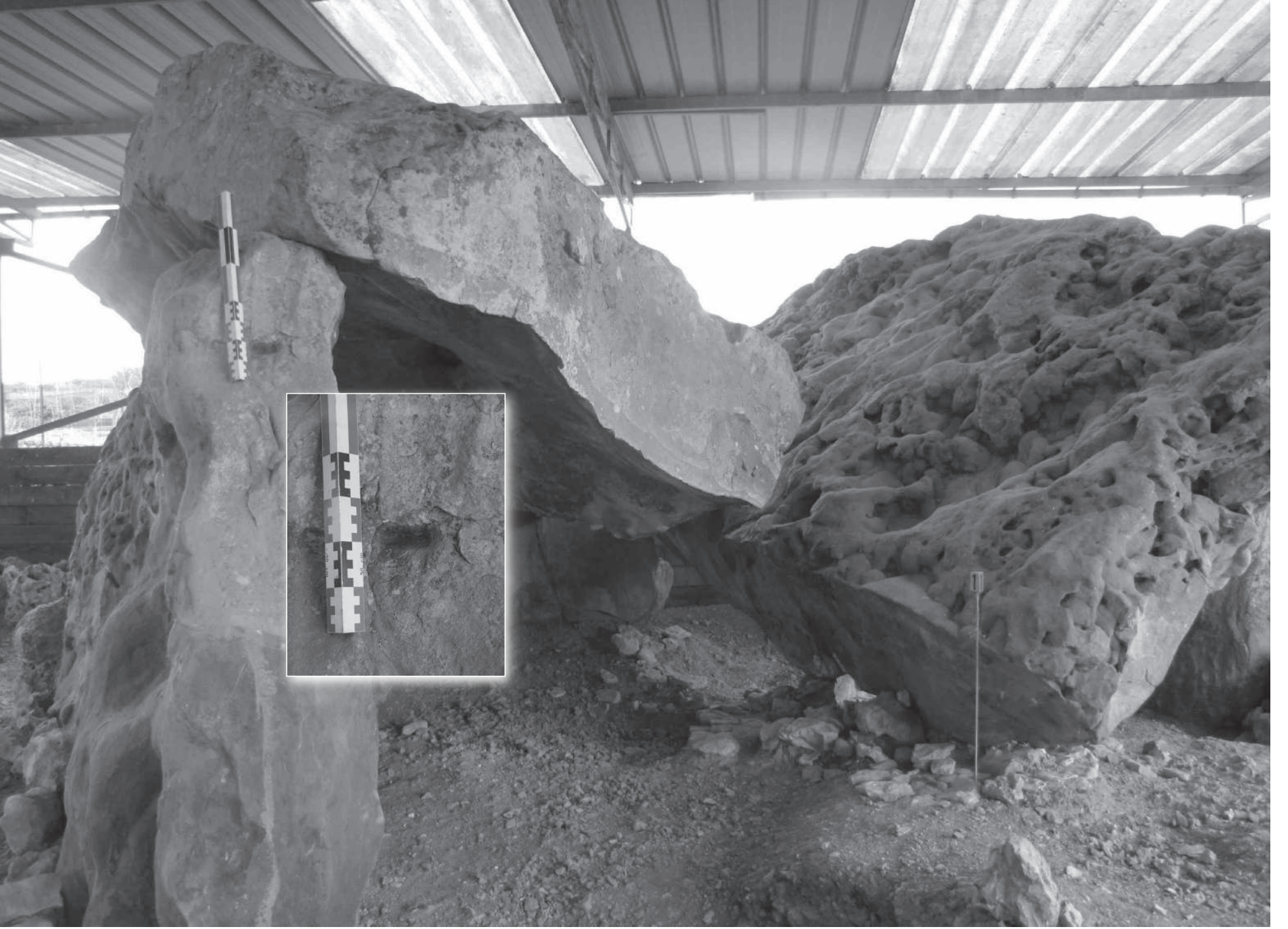

Ces deux photos illustrent la façon la plus commune de casser d'énormes blocs: cela au moyen de ce quion appelle des « boîtes de débitage »: on creusait, grosso modo en bouchardage, une petite anfractuosité destinée à recevoir un coin de bois que l'on faisait ensuite gonfler à l'eau. Cette illustration

(un plan général et un gros plan) est d'époque inconnue; elle a l'avantage d'orner l'orthostate d'un dolmen dont la dalle de couverture fut cassée dés l'époque néolithique. site ainsi qu'à Changé, usage probable aussi de «boîtes de débitage ». Quand l'opération consistait seulement à renverser une pierre levée, on retirait ses blocs de calage du côté où l'on désirait la voir tomber (c'est peut-être ce qui s'est passé à Locmariaquer?). Dans d'autre cas on est réduit à des conjectures plausibles, comme à Méréaucourt où le basculement d'une dalle de couverture paraît avoir été obtenu par un mouvement latéral lui retirant le soutien d'une partie de ses supports. Souvent, hélas, si le geste proprement dit de destruction peut être attribué sans erreur aux Néolithiques, le procédé employé reste dans l'ombre.

Il est important de savoir que l'état dans lequel nous, préhistoriens, trouvons aujourd'hui les monuments mégalithiques n'est pas un simple état d'abandon, plus ou moins suivi d'avatars divers. il s'agit bien de sites intentionnellement 
modifiés, condamnés, clos. Cette situation ne facilite pas la recherche de ce qui, il faut l'avouer, intéresse le plus: l'utilisation funéraire de ces sépulcres monumentaux et ce qu'ils représentaient, jadis, pour les groupes humains qui leur confiaient les restes de leurs membres disparus. Toutefois, ce qui n'est plus pour nous que gestes de destruction avait jadis sans doute, pour ceux qui s'y employaient, une signification des plus riches sur le plan symbolique. Faute de pouvoir accéder au sens de ces symboles, une meilleure connaissance de ces gestes, ainsi que des techniques utilisées à cette fin, représente un pas de plus dans la connaissance des populations néolithiques. 


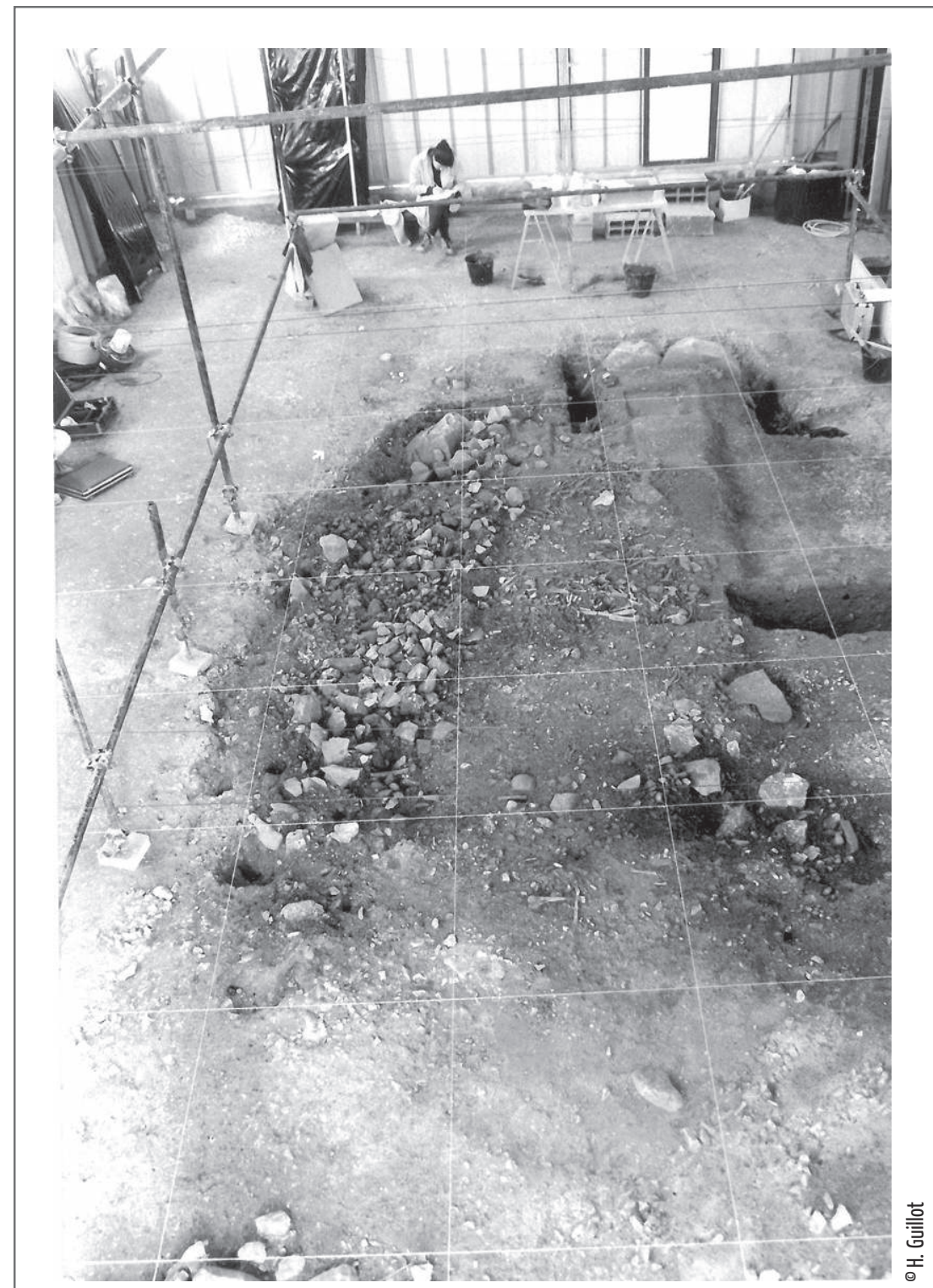

Lambeau de la dernière couche de condamnation du monument avec gros fragments de grès provenant,

pour partie, des orthostates débités par le feu. Plusieurs « remontages » entre des fragments de la couche et les orthostates ont pu être opérés.

St Sauveur (Somme), les Gros Grès (fouille et analyse : Guillot, H., Guy, H.) 


\section{흠
퐁
픙}

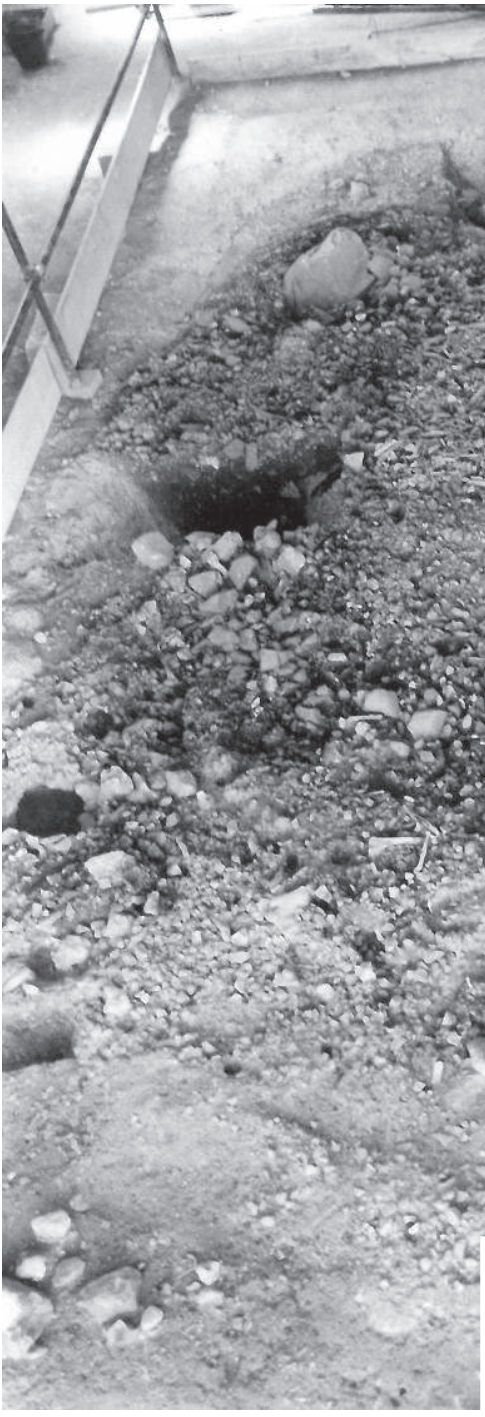

Deuxième phase de condamnation du monument. Après avoir comblé les fosses d'implantation des orthostates, une première couche de condamnation constituée de petits nodules de craie est déposée sur l'ensemble du monument, la chambre funéraire et les emplacements précédents des orthostates.

St Sauveur (Somme), les Gros Grès (fouille et analyse Guillot, H., Guy, H.)
L'orthostat du chevet a été basculé lors de la condamnation du monument. Pour ce faire, sa fosse d'implantation a été agrandie vers l'extérieur permettant sa bascule. À gauche de la photo, on distingue le bord de sa fosse d'implantation du côté de la chambre funéraire. St Sauveur (Somme), les Gros Grès (fouille et analyse : Guillot, H., Guy, H.)

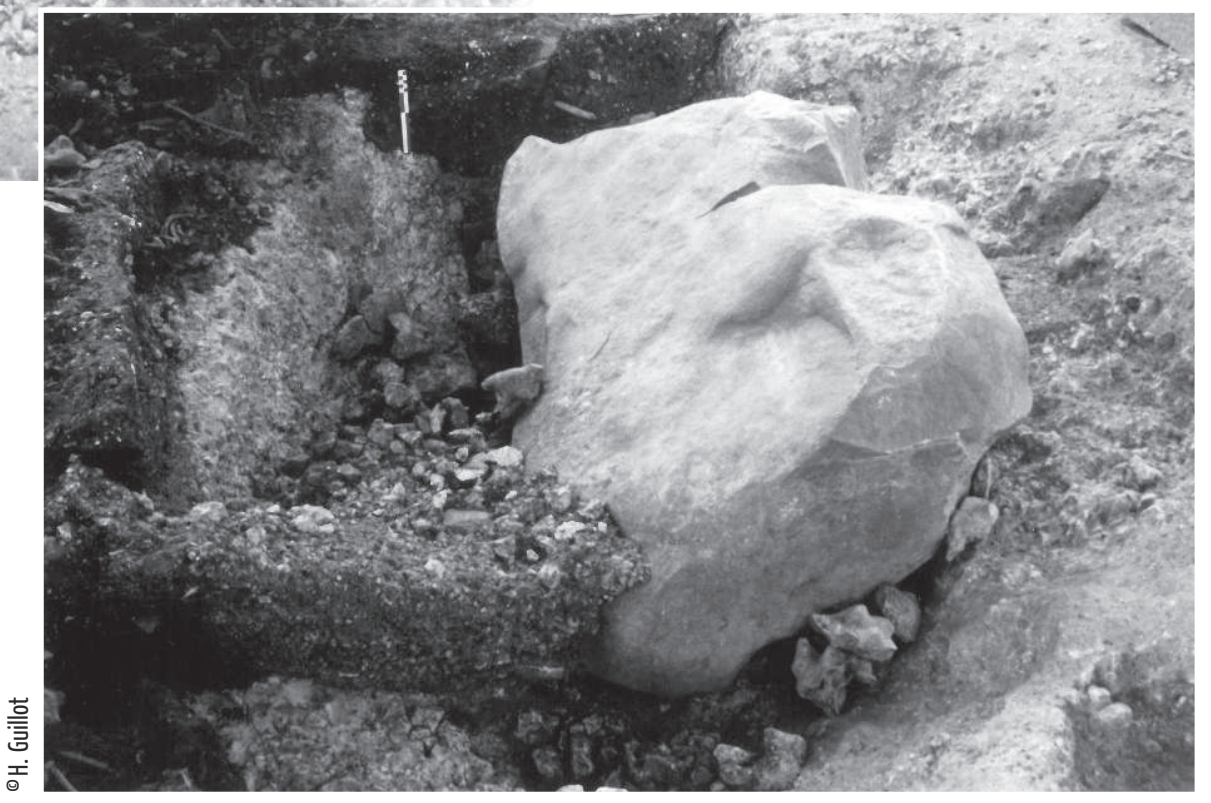




\section{RÉFÉRENCES}

Aubrey, J. 1695-97 Monumenta britannica; or miscellany of british antiquities. Vol. 1, Section 1. Templa Druidum. Oxford: MS de la Bodleian Library.

Atkinson, R.J.C. 1956 Stonehenge. London: Hamilton.

Burl, A. 1979 Prehistoric vvebury. New Haven/London: Yale University Press.

Carré, H. 1963 Le problème des dalles et de leur manutention d'après la stratigraphie, Bulletin de la Société Préhistorique Française 60 (3-4) : 186-1 90.

Coles, J. 1973 Archaeology by Experiment. London: Hutchinson University Library.

Garfitt, J. E. 1979 Moving the Stones to Stonehenge, Antiquity LIII (209) : 190-194.

Gaumé, E. L'Exploitation néolithique du granite dans le Golfe du Morbihan, ou l'épopée mégalithique des premiers granitiers du ve au III millénaire avant J.-C. à Locmariaquer. Paris: Mémoire de l'École des Hautes Études en Sciences Sociales.

Heyerdahl, T. 1958 Aku-Aku, the Secret of Easter Island. Chicago: Rand McNally.

Heizer, R.F. 1966 Ancient heavy transport, methods and achievements, Science 153: 821-830.

Jagu, D. \& Van Vliet-Lanoe B. 1991Intérêt des dépôts calcités: l'exemple des dolmens de Changé à SaintPiat (Eure-et-Loir). In Méthodes d'étude des sépultures. Compte-rendu de la table ronde des 8, 9 et 10 mai 1991. Paris: G.D.R. 742 du CNRS: 57-60.

Joussaume, R. \& Raharijaona V. 1985 Sépultures mégalithiques à Madagascar, Bulletin de la Société Préhistorique Française 82 (10-12) : 534-551.

Lafolie, C. 1819 Mémoires historiques relatifs à la fonte et à lélévation de la statue équestre de Henri Iv. Paris.

L'Helgouach, J. 1983 Les idoles qu'on abat... (ou les vicissitudes des grandes stèles de Locmariaquer), Archéologie Armoricaine 110. Vannes: Société Polymathique du Morbihan.

Leclerc, J. \& Cl. Masset 1980 Construction, remaniements et condamnation d'une sépulture collective néolithique: la Chaussée-Tirancourt (Somme). Bulletin de la Société Préhistorique Française. 72-2 : 57-64. Mohen, J.-P. 1989 Le Monde des Mégalithes. Paris: Casterman (Archives du temps).

Peek, J. 1975 Inventaire des mégalithes de la France. 4 - Région parisienne. $1^{\text {er }}$ supplément à Gallia Préhistoire. Paris: Éditions du CNRS. 


\section{RÉSUMÉ}

Construction et destruction des monuments mégalithiques. Sauf pour l'exploitation des roches en carrières, la fouille apporte peu d'indication sur la façon dont furent jadis construits les monuments mégalithiques. On est réduit à des démarches indirectes qui sont d'ailleurs suggestives, ne serait-ce que sur le plan des structures sociales concernées. La destruction de ceux d'entre eux qui étaient utilisés comme sépulcres s'est montrée riche d'enseignements. Leurs propriétaires néolithiques n'hésitaient pas, lors du passage d'une phase d'utilisation à une autre, à extraire ou à renverser des piliers, voire à faire effondrer le plafond d'une cavité. Des remaniements de plus grande ampleur intervenaient en fin d'utilisation: incendies, fractures, élimination ou au contraire apport de dalles de couverture, démontage ou érection de tumulus, etc. Décevante pour le préhistorien qui remonte difficilement, de ces ruines délibérément léguées par les Néolithiques, aux sépulcres originels, ces destructions livrent en revanche des témoignages d'ordre technique.

Construction and destruction of megalithich monuments. Exept for te working of rock in quarries, digs yield little information as how megalithic monuments used to be built. One is reduced to indirect approaches, wich are, as a matter of fact, suggestive, if only on the level of the related social structures. The destruction of those wich were utilized astombs turned out be a rich source of information. Their neolithic owners did not hesitate during the passage from one phase of utilization to anather to remove or knock down pillars, even to collapse the ceiling of an excavation. much more important alterations were made at the end of a phase: fire, fracturing, removing or on the contrary adding covering slabs, destroying or ercting a tumulus, etc. A deception for the prehistorian who is trying to go back from these ruins deliberately left by the Neolithics to the original sepulchers, these destructions nevertheless impart documentation of a technical order

\section{NOTES ET RÉFÉRENCES DE l'INTRODUCTION}

Cleuziou, S, Tosi M. (eds). 2007 In the shadow of the ancestors : the prehistoric foundations of the early Arabian civilisation in oman. muscat, Ministry of heritage and culture, $334 \mathrm{p}$.

Guillot, H., Guy, H. 1996 L'Utilisation d'un système d'information géographique (SIG) pour l'étude d'une sépulture collective. L'exemple de St Sauveur. In : Internéo 1 : 103-112.

Leroi-Gourhan, A., Bailloud, G. \& Brézillon, M. 1963 L'Hypogée II des Mournouards (Mesnil-sur-Oger, Marne). Gallia Préhistoire 5 : 23-133

Masset, C. 1993 Les Dolmens. Société néolithique et pratiques funéraires. Les sépultures collectives d’Europe occidentale. Paris : Errance : $180 \mathrm{p}$.

Munoz, O., Guy, H. 2007 Tomb 1 at Ra's Al-Jinz RJ-1 and its associated bone pits. In Cleuziou, S. \& Tosi M. (Eds). In the shadow of the ancestors: the prehistoric foundations of the early Arabian civilisation in oman. muscat, Ministry of heritage and culture : 133-136 